Relations industrielles

Industrial Relations

\title{
Pierre Verge et Gregor Murray. Le droit et les syndicats - Aspects du droit syndical québécois
}

\section{Michel Grant}

Volume 47, numéro 3, 1992

URI : https://id.erudit.org/iderudit/050799ar

DOI : https://doi.org/10.7202/050799ar

Aller au sommaire du numéro

Éditeur(s)

Département des relations industrielles de l'Université Laval

ISSN

0034-379X (imprimé)

1703-8138 (numérique)

Découvrir la revue

Citer ce compte rendu

Grant, M. (1992). Compte rendu de [Pierre Verge et Gregor Murray. Le droit et les syndicats - Aspects du droit syndical québécois]. Relations industrielles /

Industrial Relations, 47(3), 579-581. https://doi.org/10.7202/050799ar

Tous droits réservés @ C Département des relations industrielles de l'Universite Laval, 1992
Ce document est protégé par la loi sur le droit d'auteur. L’utilisation des services d'Érudit (y compris la reproduction) est assujettie à sa politique d'utilisation que vous pouvez consulter en ligne.

https://apropos.erudit.org/fr/usagers/politique-dutilisation/ 
Dans l'ensemble, l'ouvrage traite surtout des rapports collectifs du travail dans le secteur manufacturier. Décrire les relations du travail, comme le suggère le titre du livre, aurait exigé de tenir compte également de la GRH et des politiques publiques de main-d'oeuvre (Dictionnaire canadien des relations du travail).

Finalement, il y a place, même après ce livre, pour un nouvel effort visant à procurer un manuel d'introduction aux relations du travail qui soit complet et synthétique. L'excellent ouvrage sous la direction de Rodrigue Blouin, Vingt-cinq ans de pratique en relations industrielles au Québec, publié aux éditions Yvon Blais, est manifestement trop volumineux et coûteux pour remplir ce rôle. Mais il en constitue sûrement un très bon exemple. Le livre de Gérin-Lajoie est trop spécifique au cours qu'il donne à HEC (p. 2). De plus, l'absence de tout cadre théorique explicite et de toute approche historique amènera certains lecteurs et professeurs à lui substituer le livre de Boivin et Guilbault, Les relations patronales-syndicales, plus complet à cet égard et publié chez le même éditeur. Or, ces deux ouvrages couvrent grosso modo la même matière, avec des approches assez semblables. Le livre de Gérin-Lajoie a ses forces, toute la deuxième partie en fait. Le livre de Boivin et Guilbault a les siennes qu'on ne retrouve pas chez le premier auteur. Plutôt que de placer les lecteurs devant un choix pénible entre l'un ou l'autre de ces volumes, ou pire devant le choix de devoir acheter les deux volumes pour avoir un livre d'introduction encore pas tout à fait complet, l'éditeur aurait pu tenter une fusion ou trouver une autre solution à ce dilemme.

Nous sommes encore en attente d'un livre complet d'introduction aux relations du travail. Le livre de Gérin-Lajoie apporte des éléments nouveaux, valables, mais incomplets à ce projet.

Guy Bellemare

Université du Québec à Hull

Le droit et les syndicats - Aspects du droit syndical québécois, par Pierre Verge et Gregor Murray, Québec, Les Presses de l'Université Laval, 1991, 500 p., ISBN 2-7637-7257-9

Le titre du volume annonce fidèlement son contenu. Il s'agit essentiellement d'un examen du cadre juridique dans lequel s'inscrit le syndicalisme. Les auteurs ne limitent pas leur recherche doctrinale et jurisprudentielle au Code du travail et à la juridiction provinciale du travail au Québec. Les diverses dimensions du droit syndical couvert dans l'ensemble des chapitres comprennent la juridiction au niveau fédéral; ainsi les dispositions du Code canadien du travail font l'objet de l'attention soutenue des auteurs.

Cet ouvrage ne s'adresse cependant pas à des étudiants de premier cycle ou à toute personne abordant le sujet pour la première fois. Ainsi, j'entrevois difficilement comment ce livre pourrait servir dans un cours d'introduction. L'approche retenue par les auteurs présume que le lecteur possède des connaissances préalables sur les régimes existants d'accréditation et de négociation. Les étudiants gradués et les praticiens y puiseront toutefois des renseignements et des analyses utiles. Le style d'écriture pose parfois certains 
problèmes par des constructions lourdes et longues, mais cette lacune n'a pas pour effet d'appauvrir le contenu.

Comme les auteurs l'indiquent dans leur introduction, le syndicalisme est une réalité qui s'impose d'elle-même avant de devenir une construction juridique. Verge et Murray cherchent donc à voir dans quelle mesure le droit reconnaît et appréhende la réalité syndicale; ils veulent aussi identifier l'impact de ce droit sur la vie interne des organisations syndicales en examinant par exemple la conciliation entre la protection des droits individuels et les exigences de l'action collective. L'ouvrage se divise en deux grandes parties: (1) institution et (2) action.

La première partie couvre les dimensions externes et internes de l'institution syndicale. La dimension externe à laquelle les auteurs se réfèrent concerne les conditions et les composantes juridiques relatives à la représentativité syndicale, particulièrement celles concernant les conséquences de l'octroi du monopole de représentation collective des salariés au syndicat majoritaire dans l'entreprise; Verge et Murray cherchent même à identifier les fondements des quelques expériences de négociation sectorielle en distinguant les situations d'absence de représentativité sectorielle et celles où cette représentativité existe.

Les auteurs abordent ensuite toute la question juridique de l'organisation comme personne morale de même que les effets de cette personnalisation. Ils retracent les sources et le cheminement historiques de la recherche de sécurité juridique par ces organisations ouvrières. L'étude des dispositions législatives les conduit à distinguer d'une part la personnalisation directe octroyée en vertu de la Loi des syndicats professionnels, et d'autre part la personnalisation indirecte obtenue au moment de l'accréditation. Les auteurs concluent que l'évolution jurisprudentielle a conduit dans ce dernier cas à la reconnaissance générale de l'entité syndicale, même celle non constituée en corporation.

Verge et Murray s'attachent ensuite aux caractéristiques internes de l'institution syndicale. Ils tentent d'abord de localiser le pouvoir décisionnel à travers les méandres des structures tant sur le plan vertical (v.g. fédération professionnelle) que sur le plan horizontal (v.g. centrale syndicale). Ils cheminent en commençant par le syndicat local et remontent aux diverses affiliations et autres ramifications structurelles de ce dernier. L'exercice du pouvoir décisionnel doit s'articuler à l'intérieur de paramètres légaux analysés par les auteurs. Ainsi des questions comme la cotisation syndicale et l'affectation de son produit de même que les décisions liées à la négociation collective font l'objet de leur étude.

Poursuivant leur examen des dimensions internes de l'organisme syndical, les deux auteurs se penchent sur l'appartenance des membres de celui-ci. Après avoir présenté leurs réflexions relatives à l'état du droit sur l'existence et sur la portée de l'obligation d'appartenir à un syndicat, Verge et Murray analysent la légalité de dispositions concernant une telle obligation et abordent la question en fonction du concept de liberté d'association formulé par les deux Chartes. Ils se demandent s'il existe une liberté protégée de non-association. Cette partie de l'ouvrage se termirie par des considérations et par des interrogations sur le droit d'un salarié d'appartenir à un syndicat et sur le maintien de celui-ci dans son 
statut de membre; ainsi les auteurs identifient les conditions juridiques de l'exercice de l'autorité disciplinaire du syndicat, et ce jusqu'à l'expulsion d'un membre.

La seconde partie du volume porte sur les diverses formes d'action déployées par les syndicats dans la poursuite de leurs objectifs. Les auteurs consacrent un chapitre à la présentation de la double fonction syndicale, soit (1) l'action professionnelle correspondant au rôle de défense des intérêts socioéconomiques des membres et dont la négociation collective demeure la principale expression, (2) l'action politique traduisant le rôle d'agent de transformation au niveau sociétal. Afin de situer le contexte juridique et institutionnel de l'action professionnelle, les différents modèles de négociation collective sont décrits.

L'action professionnelle devient conflictuelle lorsqu'elle se manifeste ouvertement sur le terrain de la lutte économique entre patrons et syndicats. Les auteurs se demandent jusqu'où peut s'étendre la coalition des salariés et jusqu'où le droit permet au syndicat de porter le conflit. Dans ce cadre, ils examinent les conditions de la participation des salariés tant à la grève qu'aux autres actions collectives comme le piquetage et les formes de boycottage. Verge et Murray poussent plus loin dans leur analyse en cernant non seulement les responsabilités de l'association accréditée mais aussi celles des instances syndicales auxquelles cette dernière peut être affiliée. Une longue section de ce chapitre sur l'action professionnelle de type conflictuel dans les cas de conflit de droit, la négociation collective portant quant à elle sur les conflits d'intérêt entre les parties. Les auteurs se penchent sur les diverses formes qu'épouse la capacité juridique du syndicat à représenter et à défendre le salarié. Dans certaines situations, le syndicat paraitra agir au nom d'un ou plusieurs salariés individuels; dans d'autres situations, il s'identifiera à une collectivité anonyme de salariés. Si la situation personnelle d'un salarié est en cause, il faut se poser des questions non seulement sur la qualité pour agir du syndicat mais aussi sur son obligation d'agir. Lorsqu'il s'agit de l'affirmation exclusive d'un intérêt collectif par l'organisation syndicale, les auteurs distinguent les problèmes qui se posent à l'échelle de l'entreprise et ceux qui se posent à l'échelle de la profession.

Finalement, le lecteur constate que l'action politique syndicale ne se réduit pas à l'appui à un parti politique; de plus, elle peut être directe ou indirecte. En effet, cette action épouse diverses formes allant de l'affiliation à un parti politique (v.g. Nouveau Parti Démocratique) jusqu'à la participation à divers organismes étatiques et autres organismes de concertation.

Les auteurs concluent que le droit perçoit le syndicat d'abord comme un agent de négociation collective en l'investissant d'un pouvoir exclusif de représentation. L'intervention législative "conduit à privilégier l'action à dominante professionnelle par rapport à celle à caractère civique". De plus, les pratiques découlant des lois régissant les rapports collectifs du travail expriment aussi une réalité où "la finalité générale du syndicat, soit la protection de collectivités de salariés, tend à se réduire à la négociation collectiven.

Michel Grant 